\title{
The importance of the spontaneous breathing trial to keep extubated
}

\author{
Carles Subirà ${ }^{1}$, Rafael Fernández ${ }^{1,2}$ \\ ${ }^{1}$ Department of Critical Care, Althaia Xarxa Assistencial Manresa, Manresa, Spain; ${ }^{2}$ Universitat Internacional de Catalunya, CIBERES, Insituto de \\ Salud Carlos III, Madrid, Spain \\ Correspondence to: Carles Subirà, MD, PhD. Department of Critical Care, Althaia Xarxa Assistencial Universitària de Manresa, C/ Dr. Soler 1-3, \\ 08243 Manresa, Spain. Email: csubira@althaia.cat. \\ Provenance: This is an invited article commissioned by the Section Editor Dr. Guo-Wei Tu (Department of Critical Care Medicine, Zhongshan \\ Hospital, Fudan University, Shanghai, China). \\ Response to: Li J, Scott JB, Duan J, Liu K, Fink JB. More than just a screen to liberate from mechanical ventilation: treat to keep extubated? Ann \\ Transl Med 2019. doi: 10.21037/atm.2019.09.100
}

Submitted Oct 15, 2019. Accepted for publication Oct 22, 2019.

doi: 10.21037/atm.2019.10.103

View this article at: http://dx.doi.org/10.21037/atm.2019.10.103

We read with interest Dr. Fink's editorial More Than fust a Screen to Liberate from Mechanical Ventilation: Treat to Keep Extubated? We agree with the main idea: physicians must avoid reintubation because is related to an increased risk of death (1).

We appreciate its comments about our recent clinical trial (2), that merit some clarifications.

In our study, the prophylaxis with NIV or HFNC was not protocolized. Probably both were restricted to those patients in high risk of failure, being difficult to confidently interpret these results. A better picture is to calculate the rate of prophylactic NIV or HFNC among patients who were extubated after the SBT (532 patients in the PSV group and 486 patients in the T-piece group). Then, $26.7 \%$ in PSV group and $22 \%$ in T-piece group $(\mathrm{P}=0.083)$ received some prophylaxis. Moreover, a higher rate of prophylaxis in PSV group may also suggest an expected higher risk of failure. Interestingly, PSV patients showed similar postextubation failure and reintubation needs.

For further discarding any influence in the main result of our study, we performed a post-hoc analysis selecting only those patients without any prophylaxis. This new population included 433 patients in PSV group and 470 patients in the PSV group. Of them, 350 patients in the PSV group (80.8\%) and 338 patients in the T-piece group (71.9\%) were successfully extubated $(\mathrm{P}=0.002)$, suggesting that $\mathrm{NIV}$ or HFNC prophylaxis of postextubation respiratory failure, did not influence the main result of our study.
About the treatment of postextubation respiratory failure, in our study protocol, the use of NIV was not recommended, but open to the physician decision. We advise not to take any conclusion from patients treated with NIV or HFNC because we have no data to ascertain whether these patients were too sick to wait until reaching reintubation criteria, or too well, allowing for a NIV trial before going to reintubation. With the actual evidence, we remain against NIV for postextubation respiratory failure treatment because it could delay reintubation and increase mortality (3). Similarly, there are no available data about HFNC as treatment of postextubation respiratory failure. More future studies are needed to identify which strategies and treatments could reduce the needing of reintubation in postextubation respiratory failure patients.

\section{Acknowledgments}

None.

\section{Footnotes}

Conflicts of Interest: The authors have no conflicts of interest to declare.

Ethical Statement: The authors are accountable for all aspects of the work in ensuring that questions related to the accuracy or integrity of any part of the work are 
Page 2 of 2

appropriately investigated and resolved.

\section{References}

1. Jaber S, Quintard H, Cinotti R, et al. Risk factors and outcomes for airway failure versus non-airway failure in the intensive care unit: A multicenter observational study of 1514 extubation procedures. Crit Care 2018;22:236.

Cite this article as: Subirà C, Fernández R. The importance of the spontaneous breathing trial to keep extubated. Ann Transl Med 2019;7(22):705. doi: 10.21037/atm.2019.10.103
Subirà and Fernández. The role of SBT in successful extubation

2. Subirà C, Hernández G, Vázquez A, et al. Effect of pressure support vs $\mathrm{T}$-piece ventilation strategies during spontaneous breathing trials on successful extubation among patients receiving mechanical ventilation: A randomized clinical trial. JAMA 2019;321:2175-82.

3. Esteban A, Frutos-Vivar F, Ferguson ND, et al. Noninvasive Positive-Pressure Ventilation for Respiratory Failure after Extubation. N Engl J Med 2004;350:2452-60. 